\title{
ON THE BOUNDARY VALUES OF HOLOMORPHIC FUNCTIONS IN WEDGE DOMAINS
}

\author{
BY ADAM KORÁNYI ${ }^{1}$
}

Communicated by A. Zygmund, February 11, 1963

Let $R^{n}$ be real $n$-dimensional Euclidean space. We call a domain $D \subset R^{n}$ a regular cone if it is nonempty, open, convex, and such that if $y \in \bar{D}$ and $y \neq 0$ then $\lambda y \in \bar{D}$ for all $\lambda>0$ but $-y \notin \bar{D}$. Let $C^{n}=R^{n} \oplus i R^{n}$ be the complexification of $R^{n}$. In $C^{n}$ we define the wedge domain corresponding to $D$ by

$$
W_{D}=\left\{z=x+i y \mid x \in R^{n}, y \in D\right\} .
$$

The distinguished boundary of $W_{D}$ is the set $\left\{z=x+i y \mid x \in R^{n}, y=0\right\}$, i.e., just $R^{n}$.

In this paper we consider the following problem. Let $f$ be a real function on $R^{n}$ satisfying certain regularity conditions. What are the conditions for the existence of a holomorphic function $F$ on $W_{D}$ such that $f$ is the limit of $\operatorname{Re} F$ as the variable approaches the distinguished boundary?

An answer to this question was given by Hans Lewy in [2] for the case where $D$ is the positive quadrant in $R^{2}$, i.e., where $W_{D}$ is equal to the product of two half planes. We are going to generalize Lewy's result in two directions: We shall consider the case of an arbitrary wedge domain, and we shall considerably relax the regularity conditions put on $f$ and $F$ in [2]. This additional generality is made possible by our method of proof, which consists in a systematic use of Fourier transform theory.

We shall prove two theorems. Theorem 1 is the simplest formulation that can be proved by our method. Theorem 2 is of a more general nature (although it does not seem to imply Theorem 1). The basic idea of its proof is, however, the same, despite the technical complications involved by the Fourier transform theory of distributions.

We denote by $L^{1}$ and $L^{2}$ the spaces of integrable, resp. squareintegrable functions on $R^{n}$. As usual, we denote by $H^{2}\left(W_{D}\right)$ the subspace of $L^{2}$ consisting of the functions of form $\lim _{y \rightarrow 0} F_{y}$ where $F(z)$ $=F(x+i y)=F_{y}(x)$ is holomorphic in $W_{D}$ and $\left\{F_{y} \mid y \in D\right\}$ is a bounded set in $L^{2}$. Let $D^{*}$ be the dual cone of $D$, i.e., the subset of the dual space of $R^{n}$ consisting of the elements $\alpha$ such that $\langle\alpha, x\rangle>0$ for all $x \in \bar{D}$. It is known (Bochner [1]) that $f \in H^{2}\left(W_{D}\right)$ if and only if $f$ is

\footnotetext{
${ }^{1}$ Partially supported by the National Science Foundation, Contract G-24943.
} 
the Fourier transform of a function in $L^{2}$ whose support is contained in $\bar{D}^{*}$.

If $f \in L^{1}$ and $P$ is a hyperplane in $R^{n}$, we denote by $\int_{P} f$ the integral of the restriction of $f$ to $P$.

Now we can prove our first result.

THEOREM 1. Let $f$ be a real-valued function such that $f \in L^{2} \cap L^{1}$. $f$ is equal to the real part of a function in $H^{2}\left(W_{D}\right)$ if and only if $\int_{P} f=0$ for almost all those hyperplanes $P$ which have an unbounded intersection with $D$.

Proof. The Fourier transform $\hat{f}$ of $f$ is continuous and contained in $L^{2}$. Denoting by $P(\alpha, b)$ the hyperplane $\{x \mid\langle\alpha, x\rangle=b\}$, we have by Fubini's theorem,

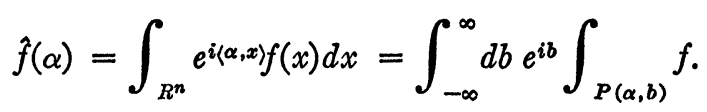

The condition of the theorem is equivalent to saying that $\int_{P(\alpha, b)} f=0$ for almost all $\alpha \notin \bar{D}^{*} \cup-\bar{D}^{*}$ and almost all $b$.

Assume that the condition is satisfied. Then it follows from (1) that the support of $\hat{f}$ is contained in $\bar{D}^{*} \cup-\bar{D}^{*}$. So $\hat{f}=\phi_{1}+\phi_{2}$, where $\phi_{1}$ and $\phi_{2}$ are real continuous functions in $L^{2}$ with support contained in $\bar{D}^{*}$ and $-\bar{D}^{*}$ respectively. Denoting by $f_{1}$ and $f_{2}$ the inverse Fourier transforms of $\phi_{1}$ and $\phi_{2}$, we have $f=f_{1}+f_{2}=\operatorname{Re}\left(f_{1}+f_{2}\right)=\operatorname{Re}\left(f_{1}+\bar{f}_{2}\right)$. By the result of Bochner mentioned above it follows that $f_{1}+\bar{f}_{2}$ $\in H^{2}\left(W_{D}\right)$.

Conversely, if $F \in H^{2}\left(W_{D}\right)$ and $f=\operatorname{Re} F=\frac{1}{2}(F+\bar{F})$ then we know that the support of $f$ is contained in $\bar{D}^{*} \cup-\bar{D}^{*}$. But (1) implies then that $\int_{P(\alpha, b)} f=0$ for all $\alpha \notin \bar{D}^{*} \cup-\bar{D}^{*}$ and almost all $b$, finishing the proof of the theorem.

One could easily prove the same result using linear submanifolds of $R^{n}$ of some fixed dimension $k(1 \leqq k \leqq n-1)$ instead of hyperplanes. In particular, the condition might involve the integral of the restriction of $f$ to straight lines. This remark applies also to the next theorem. For the sake of simplicity, however, we do not prove it in this general form; we are going to formulate it for the case of straight lines.

To state the second theorem we need some definitions. Let $\alpha$, $x \in R^{n}$. Slightly generalizing a definition of Lewy [2], we say that a function $f$ is $\left[\alpha^{0}, x^{0}\right]$-continuously summable on $R^{n}$ if the integral

$$
\int_{[\alpha, x]}|f|=\int_{-\infty}^{\infty}|f(t \alpha+x)| d t
$$


exists and converges uniformly for almost all $[\alpha, x]$ in some neighborhood of $\left[\alpha^{0}, x^{0}\right]$.

We denote by $\mathfrak{U}$ the class of real-valued functions on $R^{n}$ such that

(i) $f$ is measurable and majorized by a polynomial almost everywhere on $R^{n}$,

(ii) $f$ is $[\alpha, x]$-continuously summable for almost all $\alpha \in D, x \in R^{n}$,

(iii) for any compact set $\mathrm{A} \subset D, \int_{[\alpha, x]} f$ is bounded for almost all $\alpha \in \mathrm{A}, x \in R^{n}$.

By 3 we shall denote the class of holomorphic functions $F$ on $W_{D}$ such that for any $\epsilon>0, y \in D, t_{0}>0$ there exists a polynomial $P$ such that $\left|e^{-\epsilon t} F(x+i t y)\right| \leqq P(x)$ for all $t>t_{0}$ and all $x \in R^{n}$.

For $y \in D$ we denote by $F_{y}$ the function defined on $R^{n}$ by $F_{y}(x)$ $=F(x+i y)$. If $F \in Z$, then for all $y \in D, F_{y} \in\left(\mathcal{S}^{\prime}\right)$. (Here and in the following we make free use of the notations and terminology of Schwartz [3].) If $f \in\left(s^{\prime}\right)$ and $\lim _{y \rightarrow 0} F_{y}=f$ in $\left(\delta^{\prime}\right)$, we say that $f$ is the boundary distribution of $F$; if $f$ can be identified with a function, we call it the boundary function of $F$. We note here the fact that every $f \in \mathfrak{U}$ is contained in $\left(\mathcal{S}^{\prime}\right)$.

THEOREM 2. A function $f \in \mathfrak{U}$ is the boundary function of the real part of some function $F \in \mathbb{B}$ if and only if for almost every fixed $\alpha \in D$, $\int_{[\alpha, x]} f$ is almost everywhere equal to a constant $C_{\alpha}$ independent of $x$.

Proof. Assume that the condition is satisfied. For $M>0, \alpha \in R^{n}$ we define the measure $m_{\alpha}^{M}$ on $R^{n}$ by

$$
m_{\alpha}^{M}(\phi)=\int_{-M}^{M} \phi(t \alpha) d t
$$

for all continuous $\phi$ with compact support in $R^{n}$. We have $m_{\alpha}^{M} \in\left(\Theta_{c}\right)$, so the convolution $f * m_{\alpha}^{M}$ exists and is in $\left(s^{\prime}\right)$ for all $\alpha \in R^{n}$. We prove that $\lim _{M \rightarrow \infty} f * m_{\alpha}^{M}=C_{\alpha}$ in $\left(\mathcal{S}^{\prime}\right)$, uniformly for almost all $\alpha$ in any compact set $\mathrm{A} \subset D$.

For this we have to show that for any bounded set $B C(s)$,

$$
\lim _{M \rightarrow \infty}\left(f * m_{\alpha}^{M}\right)(\phi)=C_{\alpha} \int_{R^{n}} \phi
$$

uniformly for all $\phi \in B$ and almost all $\alpha \in \mathrm{A}$. If $B$ is bounded, there exists a rapidly decreasing function $k$ on $R^{n}$ such that $|\phi(x)|<k(x)$ for all $x \in R^{n}$ [3, II, p. 91]. If $A \subset D$ is compact, by definition of the class $\mathfrak{U}$ there exists a constant $C_{\mathrm{A}}$ such that $\left|C_{\alpha}\right| \leqq C_{\mathrm{A}}$ for $\alpha \in \mathrm{A}$. Now, given any $\epsilon>0$, we can choose a compact set $K \subset R^{n}$ such that 


$$
2 C_{\mathrm{A}} \int_{\mathfrak{C}_{K}} k(x) d x<\frac{\epsilon}{2}
$$

and $M_{0}>0$ such that

$$
\left|C_{\alpha}-\int_{-M}^{M} f(t \alpha+x) d t\right|<\frac{\epsilon}{2(\operatorname{Max} k)(\operatorname{Mes} K)}
$$

for all $M>M_{0}$ and almost all $\alpha \in \mathrm{A}, x \in K$. Now we have for all $\phi \in B, M>M_{0}$ and almost all $\alpha \in \mathrm{A}$,

$$
\left|\left(f * m_{\alpha}^{M}-C_{\alpha}\right)(\phi)\right|=\left|\int_{R^{n}}\left(\int_{-M}^{M} f(t \alpha+x) d t-C_{\alpha}\right) \phi(x) d x\right| \leqq \epsilon,
$$

as can be seen at once by splitting the integral over $R^{n}$ as $\int_{R^{n}}=\int_{\boldsymbol{K}}$ $+\int \mathfrak{e}_{K}$.

Using some fundamental properties of the Fourier transform in $\left(\mathcal{S}^{\prime}\right)$ it follows that $\hat{f} \in\left(\mathcal{S}^{\prime}\right), \hat{m}_{\alpha}^{M} \in\left(\mathcal{O}_{M}^{\prime}\right)$ and

$$
\lim _{m \rightarrow \infty} \hat{m}_{\alpha}^{M} \hat{f}=C_{\alpha} \delta
$$

in the $\left(\mathcal{S}^{\prime}\right)$-sense uniformly for almost all $\alpha \in \mathrm{A}$. (We have denoted by $\hat{f}, \hat{m}_{\alpha}^{M}$ the Fourier transforms of $f, m_{\infty}^{M}$.) It is also easy to compute $\hat{m}_{\alpha}^{M}$ explicitly; it turns out that $\hat{m}_{\alpha}^{M}(x)=D_{M}(\langle\alpha, x\rangle)$, where $D_{M}$ is the classical Dirichlet kernel.

Next we show that the support of $\hat{f}$ is contained in $\bar{D}^{*} \cup-\bar{D}^{*}$. Let $U$ be a sphere in the complement of $\bar{D}^{*} \cup-\bar{D}^{*}$. We can find a compact set $A \subset D$ such that for any $x \in U$ there exists $\alpha \in \operatorname{int}(\mathrm{A})$ satisfying $\langle\alpha, x\rangle=0$. Let $w$ be a $C^{\infty}$-function whose support is $\mathbf{A}$, and such that $w(\alpha)>0$ for all $\alpha \in \operatorname{int}(\mathrm{A})$.

In the space $\left(D^{\prime}\right)$ we have $\lim _{M \rightarrow \infty} \hat{m}_{\alpha}^{M}(x)=\delta(\langle\alpha, x\rangle)$. Therefore the limit

$$
p(x)=\lim _{M \rightarrow \infty} \int_{\mathbf{A}}^{\hat{m}_{\alpha}^{M}}(x) w(\alpha) d \alpha
$$

exists, and $p(x)>0$ for all $x \in U$. By elementary properties of the Dirichlet kernel it is also easy to see that $p \in C^{\infty}$, and that the righthand side and all its derivatives converge to $p$ and its corresponding derivatives uniformly for $x \in U$.

Now let $\phi$ be any element of (s) with support contained in $U$. Defining the measure $\mu$ on $R^{n}$ by $d \mu(\alpha)=w(\alpha) d \alpha$, we have, by the above remarks, 


$$
\lim _{M \rightarrow \infty} \int_{\mathbf{A}} \hat{m}_{\alpha}^{M} \phi d \mu=p \phi
$$

in the sense of convergence in ( $\$$ ). It follows that

$$
\lim _{M \rightarrow \infty} \hat{f}\left(\int_{\mathbf{A}} \hat{m}_{\alpha}^{M} \phi d \mu(\alpha)\right)=\hat{f}(p \phi) .
$$

Applying the generalized Fubini theorem to the tensor product distribution $f \otimes \mu$, we have

$$
\hat{f}\left(\int_{\mathbf{A}} \hat{m}_{\alpha}^{M} \phi d \mu(\alpha)\right)=\int_{\mathbf{A}} \hat{f}\left(\hat{m}_{\alpha}^{M} \phi\right) d \mu(\alpha) .
$$

Now we note that the function $\alpha \rightarrow \hat{m}_{\alpha}^{M}$ from $\mathrm{A}$ to $\left(\Theta_{M}\right)$ is continuous; hence the function $\alpha \rightarrow \hat{m}_{\alpha}^{M} \hat{f}$ from A to $\left(\mathcal{S}^{\prime}\right)$ is continuous, and consequently $\alpha \rightarrow\left(\hat{m}_{\alpha}^{M} \hat{f}\right)(\phi)$ is continuous. Furthermore,

$$
\lim _{M \rightarrow \infty}\left(\hat{m}_{\alpha}^{M} \hat{f}\right)(\phi)=\lim _{M \rightarrow \infty} \hat{f}\left(\hat{m}_{\alpha}^{M} \phi\right)=0
$$

uniformly on A, by (2). It follows that

$$
\lim _{\boldsymbol{M} \rightarrow \infty} \int_{\mathbf{A}} \hat{f}\left(\hat{m}_{\alpha}^{M} \phi\right) d \mu(\alpha)=\int_{\mathbf{A}} \lim _{M \rightarrow \infty} \hat{f}\left(\hat{m}_{\alpha}^{M} \phi\right) d \mu(\alpha)=0 .
$$

From (3), (4), (5) we have $\hat{f}(p \phi)=0$. Since $\phi$ is arbitrary, and $p(x)>0$ on $U$, it follows that $\hat{f}=0$ on $U$. Since $U$ was arbitrary we have proved that the support of $\hat{f}$ is contained in $\bar{D}^{*} \cup-\bar{D}^{*}$.

We can write $\hat{f}=\phi_{1}+\phi_{2}$, where $\phi_{1}, \phi_{2}$ are in $\left(\mathcal{S}^{\prime}\right)$ and have their support contained in $\bar{D}^{*}$ and $-\bar{D}^{*}$, respectively. Let $\phi$ be the distribution defined by $\phi(t)=\phi_{1}(t)-\phi_{2}(-t)$ (where we use the function notation as an abbreviation, to be interpreted in the obvious way), and for all $y \in D$ let $F_{y}$ be the Fourier transform of $e^{-\langle t, y\rangle} \phi(t)$. By a result of J. Lions [4, Corollary to Proposition 8], it follows that the function $F$ defined on $W_{D}$ by $F(z)=F(x+i y)=F_{y}(x)$ is in the class 3 , and using the fact that the Fourier transform is a continuous map in $\left(\mathcal{S}^{\prime}\right)$ it follows easily that $\lim _{y \rightarrow 0} \operatorname{Re} F_{y}=f$.

To prove the converse statement, let $F \in \mathbb{Z}$ and let $f \in \mathfrak{U}$ be the boundary function of $\operatorname{Re} F=\frac{1}{2}(F+\bar{F})$. By the result of Lions quoted above it follows that for all $y \in D, F_{y}$ is the Fourier transform of $e^{-\langle t, y\rangle} \phi$, where $\phi \in\left(D^{\prime}\right)$, and the support of $\phi$ is contained in $\bar{D}^{*}$ $\cup-\bar{D}^{*}$. Since $f$ is the boundary function of $\operatorname{Re} F$, it follows, using the continuity of Fourier transforms, that $\phi \in\left(\boldsymbol{s}^{\prime}\right)$ and $\hat{\phi}=f$. Now $\hat{f}$ also has its support contained in $\bar{D}^{*} \cup-\bar{D}^{*}$. 
For almost all $\alpha \in D, \lim _{M \rightarrow \infty} m_{\alpha}^{M} * f$ exists by hypothesis. Therefore $\lim _{M \rightarrow \infty} \hat{m}_{\alpha}^{M} \hat{f}$ also exists in $\left(\mathcal{S}^{\prime}\right)$. The support of this limit, as can be easily seen, is the origin; therefore it is equal to a linear combination of derivatives of $\delta$. By Fourier transformation it follows that $\lim _{M \rightarrow \infty} m_{\alpha}^{M} * f$ is a polynomial; by property (iii) of the class $\mathfrak{U}$ it is also bounded, therefore it must be equal to a constant $C_{\alpha}$. This finishes the proof of the theorem.

\section{REFERENCES}

1. S. Bochner, Group invariance of Cauchy's formula in several variables, Ann. of Math. (2) 45 (1944), 686-706.

2. H. Lewy, On the relations governing the boundary values of analytic functions of two complex variables, Comm. Pure Appl. Math. 9 (1956), 295-297.

3. L. Schwartz, Thêorie des distributions. I, II, 2nd ed., Hermann, Paris, 1957 and 1959.

4. - Transformation de Laplace des distributions, Medd. Lunds Univ. Mat. Sem., Tome Supplementaire (1952), 196-206.

University of California, Berkeley 